BULLETIN OF THE POLISH

ACADEMY OF SCIENCES

MATHEMATICS

Vol. 64, No. 2-3, 2016

\title{
On Intersections of Generic Perturbations of Definable Sets
}

\author{
by \\ Jan MYCIELSKI and Krzysztof NOWAK \\ Presented by Stanisław KWAPIEN
}

Summary. Consider an o-minimal expansion $\mathcal{R}$ of a real closed field $R$ and two definable sets $E$ and $M$. We introduce concepts of a locally transitive (abbreviated to l.t.) and a strongly locally transitive (abbreviated to s.l.t.) action of $E$ on $M$. In the former case, $M$ is supposed to be of pure dimension $m$; in the latter, both $M$ and $E$ are supposed to be of pure dimension. We treat the elements of $E$ as perturbations of the set $M$. We prove that if $E$ acts l.t. on $M$, and $A$ and $B$ are two non-empty definable subsets of $M$ of dimension $\operatorname{dim} A \leq \operatorname{dim} B<\operatorname{dim} M$, then

$$
\operatorname{dim}(\sigma(A) \cap B)<\operatorname{dim} A
$$

for a generic $\sigma$ in $E$; here $\operatorname{dim} \emptyset=-1$. And if $E$ acts s.l.t. on $M$ and $A$ and $B$ are two definable subsets of $M$, then

$$
\operatorname{dim}(\sigma(A) \cap B) \leq \max \{\operatorname{dim} A+\operatorname{dim} B-m,-1\}
$$

for a generic $\sigma$ in $E$. We give an example of a l.t. action $E$ on $M$ for which the latter conclusion of the intersection theorem fails. We also prove a theorem on the intersections of generic perturbations in terms of the exceptional set $T \subset M$ of points at which $E$ is not l.t. Finally, we provide some natural conditions which imply that $T$ is a nowhere dense subset of $M$.

1. Introduction and main results. Consider an o-minimal expansion $\mathcal{R}$ of a real closed field $R$ and two definable (with parameters) subsets $E$ and $M$ of $R^{n}$. Let $\operatorname{dim} E=e$ and assume that $M$ is of pure dimension $m$, i.e. the dimension of $M$ at each point $x \in M$ is $m$. Examples of such sets are, for instance, definable topological manifolds (possibly with boundary). In this paper we set $\operatorname{dim} \emptyset=-1$. We shall investigate continuous definable

2010 Mathematics Subject Classification: 03C64, 57N75.

Key words and phrases: intersections and perturbations of definable sets, locally transitive and strongly locally transitive actions of sets, o-minimal structures.

Received 30 September 2016.

Published online 20 October 2016. 
maps

$$
\alpha: E \times M \rightarrow M, \quad \alpha(\sigma, x)=\sigma \cdot x=\sigma(x),
$$

which we call actions of $E$ on $M$. We treat the elements of $E$ as perturbations of the set $M$. For subsets $X \subset E$ and $Y \subset M$, write

$$
X \cdot Y:=\{x \cdot y \in M: x \in X, y \in Y\} .
$$

It will be often convenient to think of the points $x \in M$ as maps from $E$ into $M$. Thus we set

$$
\alpha^{x}: E \ni \sigma \mapsto \sigma \cdot x \in M
$$

We say that $\alpha$ or $E$ is locally transitive (abbreviated to l.t.) at a point $x \in M$ if $\Omega \cdot x=\alpha^{x}(\Omega)$ is a subset of $M$ of dimension $m$ for every non-empty, open subset $\Omega$ of $E$. Of course, if $E$ is l.t. at $x$, then $e \geq m$.

REMARK 1.1. It is not difficult to prove that $E$ is l.t. at $x \in M$ iff the map $\alpha^{x}$ is generically a submersion, i.e. there exists a nowhere dense subset $F$ of $E$ such that the restriction of $\alpha^{x}$ to $E \backslash F$ is a submersion of class $\mathcal{C}^{1}$. Indeed, this follows directly from the fact that there exists a finite definable stratification of $E$ of class $\mathcal{C}^{1}$ such that $\alpha^{x}$ is a $\mathcal{C}^{1}$ map on each stratum.

The set $E$ is called l.t. on a subset $A$ of $M$ if it is l.t. at every point $x \in A$. Locally transitive actions can be characterized in terms of the rank of definable maps defined below.

Let $f: V \rightarrow W$ be a definable map between definable subsets of $R^{n}$. For any $x \in R^{n}$ and $r>0$, denote by $B(x, r)$ the ball with center $x$ and radius $r$. The function

$$
V \times(0, \infty) \ni(x, r) \mapsto \operatorname{dim} f(V \cap B(x, r)) \in \mathbb{N}
$$

is definable, because the dimension of fibers from a definable family depends definably on the parameters (cf. [1, Chap. 4]). Consequently, for a fixed $x \in V$, the function $\operatorname{dim} f(V \cap B(x, r))$ is constant for $r>0$ small enough. Its common value $r_{x} f$ near zero will be called the rank of $f$ at $x ; r_{x} f$ is a definable function of the variable $x$. It is clear that $E$ is l.t. at $x$ iff the map $\alpha^{x}$ is of constant rank $m$.

Suppose now that the definable set $E$ is also of pure dimension $e$. We say that $E$ is strongly locally transitive (abbreviated to s.l.t.) at a point $x \in M$ if the fibers of the map $\alpha^{x}$ are of dimension $\leq e-m$. An easy dimension calculus, based on the following proposition (see e.g. [1, Chap. 4, Prop. 1.5]), shows that $E$ is l.t. at $x$ if $E$ is s.l.t. at $x$.

Proposition 1.2. Let $f: V \rightarrow W$ be a definable map between non-empty definable sets. Then

$$
\begin{aligned}
& \operatorname{dim} f^{-1} f(v) \leq k \text { for all } v \in V \Rightarrow \operatorname{dim} V \leq k+\operatorname{dim} f(V), \\
& \operatorname{dim} f^{-1} f(v) \geq k \text { for all } v \in V \Rightarrow \operatorname{dim} V \geq k+\operatorname{dim} f(V) .
\end{aligned}
$$


The set $E$ is called s.l.t. on a subset $A$ of $M$ if it is s.l.t. at every point $x \in A$.

The main purpose of this paper is to prove the following two theorems on intersections of generic perturbations.

TheOrem 1.3. Suppose $M$ is a definable set of pure dimension $m$. Let $A$ and $B$ be non-empty, definable subsets of $M$. If $E$ is l.t. on $A$ and $\operatorname{dim} A \leq$ $\operatorname{dim} B<m$, then there exists a definable, nowhere dense subset $Z$ of $E$ such that

$$
\operatorname{dim}(\sigma(A) \cap B)<\operatorname{dim} A
$$

for all $\sigma \in E \backslash Z$.

TheOREM 1.4. Suppose $E$ and $M$ are definable sets of pure dimension $e$ and $m$, respectively. Let $A$ and $B$ be definable subsets of $M$. If $E$ is s.l.t. on $A$, then there exists a definable subset $Z$ of $E$ such that $\operatorname{dim} Z<e$ and

$$
\operatorname{dim}(\sigma(A) \cap B) \leq d:=\max \{\operatorname{dim} A+\operatorname{dim} B-m,-1\}
$$

for all $\sigma \in E \backslash Z$.

The above results generalize the theorem on generic intersections from [6], which treated only the case where $E$ is a definable group. Their proofs will be given in the next two sections. Section 4 gives an example of a l.t. action $E$ for which Theorem 1.4 fails. In Section 5, we define an exceptional set $T \subset M$ of points at which $E$ is not l.t. and prove a theorem on intersections of generic perturbations in terms of $T$ (Corollary 5.1). Finally, we provide some natural conditions which imply that $T$ is a nowhere dense subset of $M$ (Theorem 5.2).

Some natural examples of manifolds which act l.t. (but not s.l.t.) are the following: the set of all reflections of $\mathbb{R}^{m}$ in affine hyperplanes, the set of all rotations in $\mathbb{R}^{m}$ around affine subspaces of dimension $m-2$, the set of such rotations by a fixed non-zero angle, the counterparts of these sets in the sphere $\mathbb{S}^{m}$ and in the hyperbolic space $\mathbb{H}^{m}$, as well as non-empty open subsets of the above-mentioned sets. The paper 8 provides a study of the l.t. action of the set of rotations in $\mathbb{R}^{m}, \mathbb{S}^{m}$ and $\mathbb{H}^{m}$, and its results are applied in 4, devoted to a concept of a small set which refines the concept of a Tarski nullset.

REMARK 1.5. If $\mathcal{R}$ is a polynomially bounded, o-minimal expansion of the field $\mathbb{R}$, then smooth (i.e. of class $\mathcal{C}^{\infty}$ ) definable functions constitute a quasianalytic class, i.e. the identity principle holds: two quasianalytic functions on a connected open subset $U \subset \mathbb{R}^{m}$ coincide if so do their germs at a point $a \in U$. Hence the following characterization of local transitivity. Suppose $E$ and $M$ are connected, smooth manifolds definable in $\mathcal{R}$ and $\alpha: E \times M \rightarrow M$ is a smooth, definable map. Then $\alpha$ is l.t. at a point $x \in M$ iff the set $E \cdot x$ is a subset of $M$ of dimension $m$. For example, $\mathcal{R}$ may be an 
analytic structure $\mathbb{R}_{\mathrm{an}}$ (i.e. the expansion of the field $\mathbb{R}$ by restricted analytic functions) or, more generally, a quasianalytic structure (i.e. the expansion of the field $\mathbb{R}$ by restricted quasianalytic functions; see e.g. [9, 5, 7]).

Remark 1.6. Strong local transitivity can be expressed in terms of Remmert rank. Denote by $\operatorname{dim} A_{x}$ the dimension of a definable set $A$ at a point $x$. By the Remmert rank of a definable map $f: V \rightarrow W$ at a point $x \in V$ (cf. [3, Chap. V]) we mean the number

$$
\varrho_{x} f:=\operatorname{dim} V_{x}-\operatorname{dim} f^{-1}(f(x))_{x} .
$$

Clearly, $E$ is s.l.t. at $x$ iff the map $\alpha^{x}$ is of Remmert rank $\geq m$ everywhere on $E$.

2. Proof of Theorem 1.3. We first prove that the definable set

$$
Z:=\{\sigma \in E: \exists a \in A \exists r>0[\sigma \cdot(A \cap B(a, r)) \subset B]\}
$$

is a nowhere dense subset of $E$. Otherwise it would contain an open definable subset $U$ of $E$. By definable choice, there exist definable functions

$$
a: U \rightarrow A \text { and } r: U \rightarrow(0,1)
$$

such that

$$
\sigma \cdot(A \cap B(a(\sigma), r(\sigma))) \subset B .
$$

After shrinking the open subset $U$, we may assume that the maps $a(\sigma)$ and $r(\sigma)$ are continuous. Take any point $\sigma_{0} \in U, \varepsilon:=r\left(\sigma_{0}\right) / 3$ and a neighbourhood $U_{0} \subset U$ of $\sigma_{0}$ such that

$$
d\left(a(\sigma), a\left(\sigma_{0}\right)\right)<\varepsilon \quad \text { and } \quad r(\sigma)>2 \varepsilon \quad \text { for all } \sigma \in U_{0} ;
$$

here $d$ stands for the Euclidean distance in $R^{n}$. Then

$$
B\left(a\left(\sigma_{0}\right), \varepsilon\right) \subset B(a(\sigma), 2 \varepsilon) \subset B(a(\sigma), r(\sigma))
$$

for all $\sigma \in U_{0}$, and thus

$$
\sigma \cdot\left(A \cap B\left(a\left(\sigma_{0}\right), \varepsilon\right)\right) \subset B \quad \text { for all } \sigma \in U_{0} .
$$

In particular, we get

$$
\operatorname{dim}\left(U_{0} \cdot a\left(\sigma_{0}\right)\right)<m,
$$

which contradicts the fact that $E$ is l.t. on the set $A$. Therefore the definable set $Z$ is a nowhere dense subset of $E$, as asserted.

Consequently, we get

$$
\sigma \cdot(A \cap B(a, r)) \not \subset B
$$

for all $a \in A, r>0$ and $\sigma \in E \backslash Z$. Hence

$$
\operatorname{dim}(\sigma(A) \cap B)<\operatorname{dim} A
$$

for all $\sigma \in E \backslash Z$. Indeed, this is an immediate consequence of 
Lemma 2.1. Given definable subsets $C$ and $D$, if $\operatorname{dim}(C \cap D)=\operatorname{dim} C$, then $C \cap B(a, r) \subset D$ for some $a \in C$ and $r>0$.

This, in turn, follows directly from the existence of a finite definable cell decomposition compatible with the sets $C$ and $D$. Thus the proof of Theorem 1.3 is complete.

3. Proof of Theorem 1.4. The proof relies on dimension calculus similar to that applied in the proof of the theorem on generic intersections in [6]. We adopt the notation from that paper with the definable group $G$ replaced by the definable topological manifold $E$. Let

$$
\Delta=\Delta_{M}:=\{(x, x): x \in M\} \text { and } \pi: \Delta \rightarrow M
$$

be the diagonal and the projection onto the first factor. Then

$$
\begin{aligned}
\sigma(A) \cap B & =\pi((\sigma(A) \times B) \cap \Delta) \\
& =\pi \circ\left(\sigma \times \operatorname{Id}_{M}\right)((A \times B) \cap\{(x, \sigma(x)): x \in M\}) .
\end{aligned}
$$

Hence the sets $\sigma(A) \cap B$ and $(A \times B) \cap\{(x, \sigma(x)): x \in M\}$ are definably homeomorphic, and thus we have to find a definable, nowhere dense subset $Z$ of $E$ such that

$$
\operatorname{dim}(A \times B) \cap\{(x, \sigma(x)): x \in M\} \leq d \quad \text { for all } \sigma \in G \backslash Z .
$$

Therefore Theorem 1.4 follows immediately from the lemma below (cf. [6, p. 23]).

Lemma 3.1. The subset $Z$ of all $\sigma \in E$ such that

$$
\operatorname{dim}(A \times B) \cap\{(x, \sigma(x)): x \in M\}>d
$$

is definable and nowhere dense in $E$.

Its proof can be repeated verbatim because it requires only that the fibers

$$
\{\sigma \in E: \sigma(x)=y\}=\left(\alpha^{x}\right)^{-1}(y), \quad x \in A, y \in B,
$$

be of dimension $\leq e-m$ (in the present setting). But this is just the assumption that $E$ is s.l.t. on $A$.

4. Examples. We will show that Theorem 1.3 is asymmetric in the sense that the assumption $\operatorname{dim} A \leq \operatorname{dim} B$ is essential, even in the case where $E$ is a submanifold of a definable Lie group $G$ which acts transitively on a definable manifold $M$. In particular, in this example the action of $E$ on $M$ will be l.t. but the action of

$$
E^{-1}:=\left\{\sigma^{-1} \in G: \sigma \in E\right\}
$$

will not be l.t. on $M$. 
Let $M:=R^{2} \backslash\{(0,0)\}$ and

$$
E:=\left\{\frac{1}{1-x y}\left[\begin{array}{cc}
1 & -x \\
-y & 1
\end{array}\right] \in \mathrm{GL}(2, R): x, y \in R\right\} ;
$$

of course, the general linear group $\mathrm{GL}(2, R)$ acts transitively on $M$ and $E$ is an algebraic submanifold of $\mathrm{GL}(2, R)$. Then, by an easy calculation, for every $(a, b) \in M$ the set $E \cdot(a, b)$ contains all points $(c, d)$ with $c \neq 0$ and $d \neq 0$. Hence $E$ is 1.t. on $M$. Clearly,

$$
E^{-1}:=\left\{\left[\begin{array}{ll}
1 & x \\
y & 1
\end{array}\right] \in \mathrm{GL}(2, R): x, y \in R\right\} .
$$

Further,

$$
E^{-1} \cdot(u, 0)=\{(u, v): v \in R\}
$$

for all $u \neq 0$. Since the above set is of dimension $1, E^{-1}$ is not l.t. at $(u, 0)$.

Now let us show that the assumption $\operatorname{dim} A \leq \operatorname{dim} B$ is needed. Keep the notation of the above example. Let $A$ be the line $\{(1, t): t \in R\}$ and $B:=$ $\{(1, v)\}$. Thus $E$ is l.t. but $\operatorname{dim} A>\operatorname{dim} B$. For all $\sigma \in E$, we have $B \subset \sigma(A)$ (the equivalent relation $\sigma^{-1}(B) \subset A$ is obvious). Hence $\operatorname{dim}(\sigma(A) \cap B)=0$, while the conclusion of Theorem 1.3 would require it to be -1 , i.e. the intersection to be empty.

REMARK 4.1. Suppose that a definable group $G$ acts transitively on a definable manifold $M$ and that $E$ is a definable subset of $G$ of pure dimension. It is not difficult to check that if $E$ is s.l.t. on $M$, then $E^{-1}$ is l.t. on $M$.

When $\alpha: E \times M \rightarrow M$ is a definable map of class $\mathcal{C}^{1}$ between two definable manifolds of class $\mathcal{C}^{1}$, we call $\alpha$ a submersive action at a point $x \in M$ if the map $\alpha^{x}$ is a submersion. $E$ is called submersive on a subset $A$ of $M$ if it is submersive at every point $x \in A$. Obviously, every submersive action is s.l.t. We immediately obtain the following corollary to Theorem 1.4.

Corollary 4.2. Let $A$ and $B$ be definable subsets of $M$. If $E$ is submersive on $A$, then there exists a definable subset $Z$ of $E$ such that $\operatorname{dim} Z<e$ and

$$
\operatorname{dim}(\sigma(A) \cap B) \leq d:=\max \{\operatorname{dim} A+\operatorname{dim} B-m,-1\}
$$

for all $\sigma \in E \backslash Z$.

REMARK 4.3. This corollary follows also from an o-minimal version of the Thom transversality theorem (see e.g. 2, Chap. 3, Theorem 2.7] for the classical version) and the existence of a definable stratification.

Proposition 4.4. The weaker assumptions of Theorem 1.1 do not imply the inequality $\operatorname{dim}(\sigma(A) \cap B) \leq d$ of Theorem 2.2. 
Proof. Let $M:=\left(R^{2} \backslash\{(0,0)\}\right) \times R$ and

$$
E:=\left\{\frac{1}{x_{1}^{2}-x_{2}^{2}}\left[\begin{array}{ccc}
x_{1} & -x_{2} & 0 \\
-x_{2} & x_{1} & 0 \\
x_{2} x_{3} & -x_{1} x_{3} & x_{1}^{2}-x_{2}^{2}
\end{array}\right] \in \operatorname{GL}(3, R)\right\} ;
$$

of course, $E$ is an algebraic submanifold of $\operatorname{GL}(3, R)$. Then, by an easy calculation, for every $a=\left(a_{1}, a_{2}, a_{3}\right) \in M$ the set $E \cdot a$ contains all points $b=\left(b_{1}, b_{2}, b_{3}\right)$ with $b_{1} \neq 0, b_{2} \neq 0, b_{1}^{2}-b_{2}^{2} \neq 0$ and $a_{2} b_{1}-a_{1} b_{2} \neq 0$. Hence $E$ is l.t. on $M$. Clearly,

$$
E^{-1}:=\left\{\left[\begin{array}{ccc}
x_{1} & x_{2} & 0 \\
x_{2} & x_{1} & 0 \\
0 & x_{3} & 1
\end{array}\right] \in \mathrm{GL}(3, R)\right\},
$$

$E^{-1}$ is not l.t. on $M$ and thus $E$ is not s.l.t. on $M$.

Let $A$ be the circle

$$
A:=\left\{a \in M: a_{1}^{2}+a_{2}^{2}=1, a_{3}=1\right\}
$$

and let $B$ be the line

$$
B:=\left\{b \in M: b_{2}=0, b_{3}=1\right\} .
$$

Then $d=\max \{\operatorname{dim} A+\operatorname{dim} B-m,-1\}=-1$.

Observe now that if $b=\left(b_{1}, 0,1\right) \in B$ and

$$
\sigma^{-1}:=\left[\begin{array}{ccc}
x_{1} & x_{2} & 0 \\
x_{2} & x_{1} & 0 \\
0 & x_{3} & 1
\end{array}\right] \in E^{-1}
$$

then $\sigma^{-1}(b)=\left(b_{1} x_{1}, b_{1} x_{2}, 1\right)$. Hence $\sigma^{-1}(B)$ is the line through the points $(0,0,1)$ and $\left(x_{1}, x_{2}, 1\right)$. Therefore $A \cap \sigma^{-1}(B)$ is a two-point set. Hence $\operatorname{dim}(\sigma(A) \cap B)=0>d$ for every $\sigma \in E$, contrary to the inequality of Theorem 1.2.

5. Exceptional set of the action. Consider further an action of a definable set $E$ on a definable set $M$ of pure dimension $m$. By the exceptional subset $T$ of $M$ with respect to the action $E$ we mean the definable set of all points $x \in M$ at which $E$ is not l.t., i.e. of those $x \in M$ for which $U \cdot x$ is of dimension $<m$ for some non-empty, open subset $U$ of $E$. Thus the assumption of Theorem 1.3 says that $A \cap T=\emptyset$. But this can be replaced by the weaker assumption $\operatorname{dim}(A \cap T)<\operatorname{dim} A$ :

Corollary 5.1. If $A$ and $B$ are non-empty, definable subsets of $M$ such that

$$
\operatorname{dim} A \leq \operatorname{dim} B<m \quad \text { and } \quad \operatorname{dim}(A \cap T)<\operatorname{dim} A,
$$


then there exists a definable, nowhere dense subset $Z$ of $E$ such that

$$
\operatorname{dim}(\sigma(A) \cap B)<\operatorname{dim} A \quad \text { for all } \sigma \in E \backslash Z .
$$

Proof. Subtract $T$ from $A$ and apply Theorem 1.3 .

The smaller the dimension of $T$, the larger the class of pairs $A, B$ for which Theorem 1.3 and Corollary 5.1 apply. Now we are going to provide some conditions under which $T$ is of dimension $<m$. For a subset $F$ of $E$ set

$$
M(F):=\{x \in M: \operatorname{dim}(F \cdot x)<m\} .
$$

Then

$$
F_{1} \subset F_{2} \Rightarrow M\left(F_{1}\right) \supset M\left(F_{2}\right)
$$

and

$$
M\left(F_{1} \cup F_{2}\right)=M\left(F_{1}\right) \cap M\left(F_{2}\right) .
$$

Obviously, if $F$ is a definable set, so is $M(F)$. Further, we get

$$
T=\bigcup_{U \subset E \text { open }} M(U)=\bigcup_{\sigma \in E, r>0} M(E \cap B(\sigma, r)) .
$$

This gives rise to the following definition. We call an element $\sigma \in E$ a singular perturbation with respect to $E$ if

$$
\operatorname{dim} M(E \cap B(\sigma, r))=m \quad \text { for some } r>0 .
$$

The set of all singular perturbations with respect to $E$ is an open definable subset of $E$. We call its closure $E_{s}$ the singular locus of $E$. The complement $E_{t}:=E \backslash E_{s}$ is an open definable subset of $E$, called the tame locus of $E$. It is not difficult to check that $E_{t}$ has no singular perturbation with respect to $E_{t}$.

THEOREM 5.2. If $E$ has no singular perturbation with respect to $E$, then the exceptional set $T$ is a subset of $M$ of dimension $<m$.

Proof. Towards a contradiction, suppose that $T$ is of dimension $m$ and thus contains an open definable subset $\Omega$ of $M$. By definable choice, there are definable functions

$$
c: \Omega \rightarrow E \quad \text { and } \quad r: \Omega \rightarrow(0, \infty) \subset R
$$

such that $x \in M(E \cap B(c(x), r(x))$ for all $x \in \Omega$. We may assume, after shrinking $\Omega$, that the functions $c$ and $r$ are continuous. Take a point $x_{0} \in \Omega$ and a neighbourhood $\Omega_{0}$ of $x_{0}$ such that

$$
d\left(c(x), c\left(x_{0}\right)<r_{0} / 3 \quad \text { and } \quad r(x)>2 r_{0} / 3 \quad \text { for all } x \in \Omega_{0},\right.
$$

where $r_{0}:=r\left(x_{0}\right)$. Then

$$
B(c(x), r(x)) \supset B\left(c\left(x_{0}\right), r_{0} / 3\right) \quad \text { for all } x \in \Omega_{0},
$$


and

$$
x \in M(E \cap B(c(x), r(x))) \subset M\left(E \cap B\left(c\left(x_{0}\right), r_{0} / 3\right)\right) \quad \text { for all } x \in \Omega_{0} .
$$

Hence

$$
\Omega_{0} \subset M\left(E \cap B\left(c\left(x_{0}\right), r_{0} / 3\right)\right),
$$

and thus $c\left(x_{0}\right) \in E$ is a singular perturbation with respect to $E$, contrary to the assumption. This finishes the proof.

REMARK 5.3. Assume that $\mathcal{R}$ is a polynomially bounded, o-minimal expansion of the field $\mathbb{R}$, and that $E$ and $M$ are connected, smooth, definable manifolds. It follows from the identity principle for quasianalytic functions (cf. Remark 1.5) that $M(U)=M(E)$ for any non-empty, open subset $U$ of $E$. Further, $M(E)$ is a definable quasianalytic subset of $M$, and hence so is the exceptional set $T=M(E)$. Thus, unlike in the general settings, $T$ is either a closed, nowhere dense subset of $M$ or the whole manifold $M$.

\section{References}

[1] L. van den Dries, Tame Topology and O-minimal Structures, Cambridge Univ. Press, 1998.

[2] M. W. Hirsch, Differential Topology, Springer, New York, 1976.

[3] S. Łojasiewicz, Introduction to Complex Analytic Geometry, Birkhäuser, Basel, 1991.

[4] J. Mycielski and G. Tomkowicz, On small subsets in Euclidean spaces, Bull. Polish Acad. Sci. Math., to appear.

[5] K. J. Nowak, Quantifier elimination, valuation property and preparation theorem in quasianalytic geometry via transformation to normal crossings, Ann. Polon. Math. 96 (2009), 247-282.

[6] K. J. Nowak, A theorem on generic intersections in an o-minimal structure, Fund. Math. 227 (2014), 21-25.

[7] K. J. Nowak, Quantifier elimination in quasianalytic structures via non-standard analysis, Ann. Polon. Math. 114 (2015), 235-267.

[8] K. J. Nowak and G. Tomkowicz, Intersection of generic rotations in some classical spaces, Bull. Polish Acad. Sci. Math. 64 (2016), 105-107.

[9] J.-P. Rolin, P. Speissegger and A. J. Wilkie, Quasianalytic Denjoy-Carleman classes and o-minimality, J. Amer. Math. Soc. 16 (2003), 751-777.

Jan Mycielski

Department of Mathematics

University of Colorado

Boulder, CO 80309-0395, U.S.A.

E-mail: jmyciel@euclid.colorado.edu
Krzysztof Nowak

Institute of Mathematics Faculty of Mathematics and Computer Science Jagiellonian University Łojasiewicza 6 30-348 Kraków, Poland E-mail: nowak@im.uj.edu.pl 
